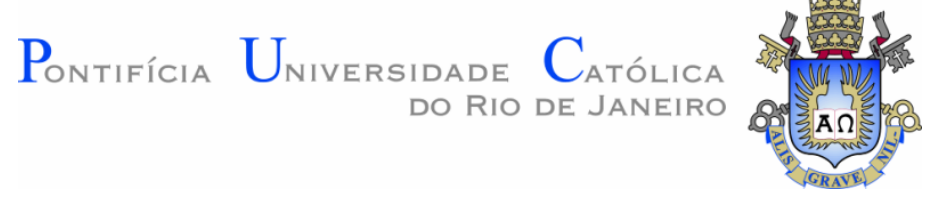

Juan José Cruz Villanueva

Estudo experimental da combustão turbulenta de sprays de etanol usando PLIF-OH, PIV e Shadowgraphy

Dissertação de Mestrado

Dissertação apresentada ao Programa de Pósgraduação em Engenharia Mecânica da PUC-Rio como requisito parcial para obtenção do grau de Mestre em Engenharia Mecânica.

Orientador: Prof. Luís Fernando Figueira da Silva

Rio de Janeiro

Abril de 2013 


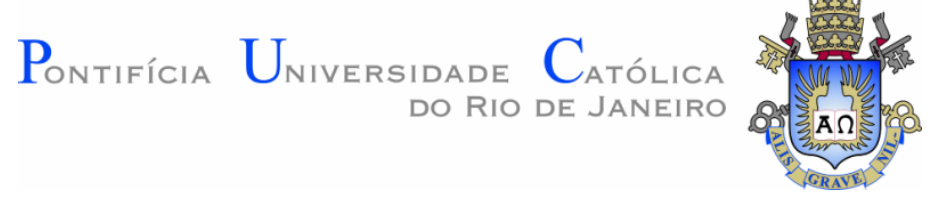

Juan José Cruz Villanueva

\title{
Estudo experimental da combustão turbulenta de sprays de etanol usando PLIF-OH, PIV e Shadowgraphy
}

\begin{abstract}
Dissertação apresentada como requisito parcial para obtenção do grau de Mestre pelo Programa de PósGraduação em Engenharia Mecânica do Centro Técnico Cientifico da PUC-Rio. Aprovada pela Comissão Examinadora abaixo assinada.
\end{abstract}

Prof. Luís Fernando Figueira da Silva

Orientador

Pontifícia Universidade Católica Do Rio De Janeiro

Prof. Amir Antônio Martins de Oliveira Júnior Universidade Federal de Santa Catarina

Prof. Guenther Carlos Krieger Filho Universidade de são Paulo

Prof. Luís Fernando Alzuguir Azevedo Pontifícia Universidade Católica Do Rio De Janeiro

Prof. José Eugenio Leal Coordenador Setorial do Centro Técnico Cientifico - PUC-Rio

Rio de Janeiro, 18 de abril de 2013 
Todos os direitos reservados. É proibida a reprodução total ou parcial do trabalho sem autorização da universidade, do autor e do orientador.

\section{Juan José Cruz Villanueva}

Graduou-se em Engenharia Mecânica na Universidade Nacional de Trujillo - UNT do Perú em 2009. Possui experiência profissional como Engenheiro de Mecânico na área de manutenção em diversas empresas peruanas, na área pesqueira e mineração.

Ficha Catalográfica

Villanueva, Juan José Cruz

Estudo experimental da combustão turbulenta de sprays de etanol usando PLIF-OH, PIV e Shadowgraphy / Juan José Cruz Villanueva; orientador: Luís Fernando Figueira da Silva. - 2013.

135 f. : il. (color.) ; $30 \mathrm{~cm}$

Dissertação (mestrado)-Pontifícia Universidade Católica do Rio de Janeiro, Departamento de Engenharia Mecânica, 2013.

Inclui bibliografia

1. Engenharia mecânica - Teses. 2. Fluorescência induzida por laser. 3. Velocímetria por imagem de partículas. 4. Turbulência. I. Silva, Luís Fernando Figueira da. II. Pontifícia Universidade Católica do Rio de Janeiro. Departamento de Enaenharia Mecânica. III. Título. 
Aos meus pais. 


\section{Agradecimentos}

Ao meu orientador Professor Luís Fernando Figueira da Silva pelo apoio, confiança, estímulo e dedicação para a realização deste trabalho.

Ao Professor Guenther Carlos Krieger Filho pela confiança e auxílios concedidos sem os quais este trabalho não poderia ter sido realizado.

Aos meus pais, pelo apoio incondicional, atenção e carinho em toda minha vida.

A CAPES, e à PUC-Rio, pelos auxílios concedidos, sem os quais este trabalho não poderia ter sido realizado.

À PETROBRAS pelo suporte financeiro destinado à infraestrutura do laboratório.

Aos meus colegas da PUC-Rio. 


\section{Resumo}

Cruz Villanueva, Juan José; Figueira da Silva, Luís Fernando. Estudo experimental da combustão turbulenta de sprays de etanol usando PLIF-OH, PIV e Shadowgraphy. Rio de Janeiro, 2013. 135p. Dissertação de Mestrado - Departamento de Engenharia Mecânica, Pontifícia Universidade Católica do Rio de Janeiro.

O presente trabalho apresenta uma análise experimental da combustão turbulenta de sprays de etanol, mediante o uso de técnicas de diagnóstico laser, em queimadores tipo obstáculo. São empregadas a fluorescência induzida por plano laser (PLIF) do radical hidroxila $(\mathrm{OH})$, para mapear a frente de chama, a velocimetria por imagens de partículas (PIV), para determinar o campo de velocidades das gotas do spray e "Shadowgraphy", para obter o diâmetro e velocidade de gota. Uma caracterização da estrutura do escoamento de ar a jusante do corpo rombudo é realizada com PIV estéreo para diferentes números de Reynolds. Os resultados mostram uma similitude do escoamento na zona de recirculação. As maiores flutuações turbulentas de velocidades são encontradas na região de vórtice e indicam anisotropia no tensor de Reynolds. Os resultados de "Shadowgraphy" revelam que as gotas do spray não são perfeitamente esféricas em regiões perto do atomizador. O diâmetro médio Sauter (SMD) foi medido em varias posições na região de recirculação. As gotas de maior diâmetro apresentaram as maiores velocidades e as pequenas são ligeiramente desviadas pela zona de recirculação nas regiões mais afastadas da linha central. Os experimentos reativos realizados com diversos valores de vazão de etanol e ar indicam que a frente de chama é descolada do queimador, sua forma é determinada principalmente pela vazão de etanol e a intensidade de luminescência é aumentada com a velocidade do ar. Em alguns casos o escoamento de ar muda a forma do spray. Uma quantidade de gotas apreciável sempre consegue atravessar a frente de chama. O campo de velocidade das gotas é influenciado pelo incremento da velocidade do escoamento anular de ar.

\section{Palavras-chave}

Fluorescência induzida por laser; velocímetria por imagem de partículas; turbulência. 


\section{Abstract}

Cruz Villanueva, Juan José; Figueira da Silva, Luís Fernando (Advisor). Experimental study of the turbulent combustion of ethanol sprays using OH-PLIF, PIV and Shadowgraphy. Rio de Janeiro, 2013. 135p. MSc. Dissertation - Departamento de Engenharia Mecânica, Pontifícia Universidade Católica do Rio de Janeiro.

This work presents an experimental analysis of turbulent combustion of ethanol sprays through the use of laser diagnostic techniques in a bluff-body burner. Are employed the planar laser-induced fluorescence (PLIF) of the hydroxyl $(\mathrm{OH})$, to map the flame front, the particle image velocimetry (PIV) to determine the velocity field of the spray droplets and "Shadowgraphy" to obtain the droplet diameter and velocity. A characterization of the structure of the air flow downstream of the bluff-body is performed with stereo PIV at different Reynolds numbers. The results evidence flow similarity in the recirculation zone. The largest turbulent velocity fluctuations are found in the vortex region, which implies the anisotropy of the Reynolds stresses. The results of "Shadowgraphy" indicate that the spray droplets are not perfectly spherical near the atomizer. The evolution of Sauter mean diameter (SMD) is measured at various positions at the recirculation region. The largest diameter droplets have the highest velocity and the smaller are slightly deviated by recirculation zone in the far furthest from the centerline. The reactive experiments are performed with different ethanol and air flow rates and indicate that the flame front is detached from the burner, the shape is determined by the ethanol flow rate and the luminescence intensity increases with the air velocity. In some case the air flow changes the shape of the spray. An appreciable number of droplets always passe through the flame front. The velocity of the droplets is influenced by the increase of speed air velocity.

\section{Keywords}

Laser induced fluorescence; particle image velocimetry; turbulence. 


\section{Sumário}

1. Introdução 22

1.1. Objetivos 25

1.2. Organização do manuscrito 26

2. Revisão Bibliográfica $\quad 27$

2.1. Aplicação do diagnóstico laser na combustão de sprays 27

2.1.1. Técnicas laser Mie, LIF e PIV aplicadas a estudos de $\begin{array}{ll}\text { combustão em sprays } & 27\end{array}$

2.1.2. Analise do spray mediante a técnica Shadowgraphy 34

2.2. Considerações finais 42

3. Metodologia Experimental 44

3.1. Queimador tipo Obstáculo (bluff-body) 44

3.2. Sistema de alimentação de ar e combustível 46

3.2.1. Bico atomizador de combustível 47

3.2.2. Propriedades do combustível empregado 47

3.3. Fluorescência induzida por plano laser (PLIF) 48

3.3.1. Fundamentos da técnica 48

3.3.2. Sistema de medição de PLIF 51

3.4. Velocimetria por imagens de partículas (PIV) 53

3.4.1. Velocímetria por imagens estereoscópicas de partículas (PIV estéreo) 54

3.4.2. Análise das imagens de partículas 56

3.4.3. Tipos de partículas traçadoras 58

3.5. Sistema de captura de imagens $\quad 59$

3.6. "Shadowgraphy" 61

3.6.1. Aquisição e processamento das imagens 63

3.6.2. Aquisição e processamento das imagens 63

3.6.3. Analise dos resultados 66 
3.6.3.2. Correção de borda 68

3.6.3.3. Correção da quantidade de gotas e resultados estatísticos $\quad 69$

3.6.3.4. Velocidade das gotas 70

4. Análise dos Resultados e Discussões 71

4.1. Escoamento de ar na esteira do obstáculo. 71

Escoamento médio e tensores de Reynolds. 72

Estrutura das propriedades turbulentas derivadas. 79

Estrutura anisotrópica turbulenta do escoamento de ar. 82

4.2. Diâmetro e velocidade de gotas de um spray de etanol na presença de ar co-corrente. $\quad 85$

4.2.1. Estrutura de desintegração do filme líquido. 86

4.2.2. Distribuição do diâmetro SMD do spray. 88

4.2.3. Distribuição do diâmetro e velocidades de gotas ao longo da linha central. $\quad 90$

4.2.4. Distribuição do diâmetro e velocidades de gotas para os casos $\boldsymbol{B}$ e $\boldsymbol{E}$

4.3. Combustão de chamas spray de etanol na presença de ar cocorrente 96

4.3.1. Estrutura global de chama por visualização direita 96

4.3.2. Comparação das imagens instantâneas de PLIF-OH e do espalhamento Mie

4.3.3. Comparação das imagens médias de PLIF-OH e do espalhamento Mie

104

4.3.4. Comparação dos campos médios de velocidade de gotas 108

5. Conclusões e Sugestões

5.1. Conclusões e contribuições 113

5.2. Sugestões para trabalhos futuros 116

6. Referências Bibliográficas 
Apêndice $B$

Apêndice $C$ 


\section{Lista de figuras}

Figura 1 - Diferentes estruturas de atomização. Imagens Mie do spray multiponto de etanol sob várias condições de temperatura e pressão ambiente. Pressão de injeção do etanol $\boldsymbol{P i n j}=\mathbf{5} \mathbf{M P a}$ [13].

Figura 2 - Razão de LIF/Mie utilizando imagens de traçadores. (a) Acetona, (b) Coumarin 47, (c) Tolueno. As imagens representam a media de 1000 imagens [14].

Figura 3 - Chama spray de etanol (a) Imagem da chama, (b) Campo de temperatura da fase liquida obtida da media de 600 imagens [15], (c) Perfis de temperatura [16], vazão de 0,035 l/min de etanol preaquecido a $45^{\circ} \mathrm{C}$ e uma velocidade do ar anular de $0,32 \mathrm{~m} / \mathrm{s}$.

Figura 4 - Distribuição quantitativa do $n$-hexano medido por LIEF para um injetor swirl de alta pressão. (a) Caso de injeção em condições de pressão e temperatura ambiente, (b) Caso de injeção do $n$-hexano a $313 \mathrm{~K}$ em condições de câmara pressurizada e aquecida [17].

Figura 5 - Imagens instantâneas do espalhamento Mie e fluorescência do radical $\mathrm{OH}$ numa chama spray de etanol. Media das imagens do espalhamento Mie e LIF-OH superposta [9].

Figura 6 - Evolução do diâmetro médio no tempo após inicio da injeção, para quatro posições sobre o eixo de pulverização.

Pinjeção = 6 Mpa e Pcamará = 2 Mpa, diâmetro do bico $d=\mathbf{2 m m}[19]$.

Figura 7 - Evolução temporal: (a) Diâmetro médio, (b) Velocidade das gotas. Três diferentes pressões de injeção $p \boldsymbol{i n j}=$ 6, 7, 8 MPa [20].

Figura 8 - Distribuição do diâmetro SMD (a) num plano horizontal $4 \mathrm{~cm}$ a jusante do bico, (b) Perfis do diâmetro SMD medidos ao 
longo de uma linha horizontal central localizada $4 \mathbf{c m}$ a jusante da saída do bico [21].

Figura 9 - Distribuição do tamanho de gota de etanol (a) variação temporal do diâmetro SMD, (b) 6 ms após o inicio da injeção e (c) $8 \mathrm{~ms}$ após o início da injeção. Pressão de injeção de 0,6 MPa e $100 \mathrm{~mm}$ acima do bico [22].

Figura 10 - Estudo de um spray transiente PFI (a) Centricidade das gotas, (b) Diferença entre o valor do SMD quando todas as gotas e só as gotas com centricidade $\boldsymbol{c}>\mathbf{0 , 7}$, (c) Velocidade das gotas, (d) Número de Weber das gotas. Pinj = 0,25 MPa, a $50 \mathrm{~mm}$ acima do bico e $\mathbf{5} \boldsymbol{m s}$ após do inicio da injeção [22].

Figura 11 - Estudo de um spray efervescente de água. (a) PDF da centricidade de gota a $\mathbf{z}=\mathbf{4 0} \mathbf{m m}$ (b) Discretização de imagens de gotas por um sensor CCD (c) Comparação da velocidade axial medida por Estéreo PIV e "Shadowgraphy" no eixo de simetria. $d=0,8 \mathrm{~mm}[24]$.

Figura 12 - Estudo de um spray de água. (a) Espalhamento Mie do spray sobreposto ao campo de velocidade. Medidas realizadas no ponto $40 \mathrm{~mm}$ do injetor (b) Distribuição do diâmetro de gotas e (c) Distribuição de velocidade. Pressão de injeção de 6 bar [25].

Figura 13 - Corte transversal do queimador mostrando suas principais características.

Figura 14 - Sistema de alimentação de combustível e ar.

Figura 15 - Morfologia de cone oco do spray utilizado [29].

Figura 16 - Diagrama de níveis de energia para um processo de fluorescência induzida [34].

Figura 17 - Componentes principais da montagem experimental de PLIF. Adaptado de [38].

Figura 18 - Espectro de emissão do corante Coumarin 153 diluído em etanol, para uma excitação de 355 nm. 
Figura 20 - Arranjo PIV estereoscópico (a) Principais componentes do sistema, (b) Deslocamento angular das câmaras. Adaptado de $[44,46]$.

Figura 21 - Arranjo PIV estereoscópico (a) Geometria de visualização no plano superior, (b) Calibração das câmaras. Adaptado de [45,44].

Figura 22 - Método de correlação cruzada para determinar a velocidade numa janela de interrogação. Adaptado de [44].

Figura 23 - Secção transversal de uma câmara ICCD acoplada à lente. As tensões de operação são indicadas. Adaptado de [52].

Figura 24 - Arranjo experimental da técnica "Shadowgraphy".

Adaptado de [55].

Figura 25 - Montagem experimental empregada para medição da distribuição de tamanho de gotas e velocidades com Shadowgraphy.

Figura 26 - Sequencia de processamento: (a) Imagem de referencia, (b) Imagem invertida, (c) Segmentação global, (d) resultado final.

Figura 27 - Perfil de intensidade da imagem mostrando os níveis umbrais empregados na primeira e segunda segmentação.

Adaptado de [61].

Figura 28 - Detalhe da placa de calibração DOF, os círculos menores $(\mathbf{1 0} \boldsymbol{\mu m})$ ficam fora de foco mais rapidamente que os maiores $(\mathbf{2 0 0} \boldsymbol{\mu m})$.

Figura 29 - Profundidade máxima do campo por classe de tamanho e relação linear da profundidade do campo como função do diâmetro.

Figura 30 - Dependência do campo de visão efetivo com o diâmetro de partículas [55].

Figura 31 - Janela de interrogação para determinar as velocidades das gotas [55].

Figura 32 - Campos componente longitudinal da velocidade media $\boldsymbol{V} \boldsymbol{y}$, linhas de corrente e vetores velocidade para (a) caso $\mathbf{1}$, (b) caso 2 e (c) caso 3, (d) Estrutura do escoamento. 
Figura 33 - Evolução longitudinal da componente longitudinal da velocidade media $V y$ para (a) linha $x=-19,7 \mathrm{~mm}$ que passa pelo centro do vórtice e (b) linha $x=-2,5 \mathrm{~mm}$.

Figura 34 - Evolução transversal das componentes adimensionais de velocidade média no caso 3 para diferentes alturas acima do obstáculo (a) $\boldsymbol{V} \boldsymbol{x} \boldsymbol{V} \boldsymbol{y} \_\boldsymbol{m a x}$, (b) VyVy_max e (c) VzV $\boldsymbol{V} y_{-} \boldsymbol{m a x}$, (d) Linhas de corrente, vetores velocidade e campo da componente transversal de velocidade $\boldsymbol{V} \boldsymbol{x}$.

Figura 35 - Evolução das componentes do tensor de Reynolds na direção $(\mathrm{x})$ no caso 3 para diferentes alturas acima do obstáculo (a) $R x x V y \_\max 2$, (b) RyyVy_max2, (c) RzzVy_max2 e (d) RxyVy_max2.

Figura 36 - Evolução das componentes do tensor de Reynolds na direção (x) no caso 3 para diferentes alturas acima do obstáculo (a) $R x z V y \_m a x 2$, (b) RyzVy_max2.

Figura 37 - Componentes do tensor taxa de deformação (a) $\boldsymbol{S} \boldsymbol{x} \boldsymbol{x}$, (b) $\boldsymbol{S y y}$, (c) $\boldsymbol{S} x \boldsymbol{y}$ e (d) taxa de rotação $\boldsymbol{W} \boldsymbol{x y}$.

Figura 38 - Distribuições tentativas de viscosidade turbulenta aplicando a hipóteses de Boussinesq (a) $\boldsymbol{v} \boldsymbol{t}_{-} \boldsymbol{x} \boldsymbol{x}$, (b) $\boldsymbol{v} \boldsymbol{t}_{-} \boldsymbol{y} \boldsymbol{y}$ e (c) $v \boldsymbol{v}$ xyy. Valores válidos apenas entre 0,001 e 0,015.

Figura 39 - Triângulo das invariantes anisotrópicas III e II do tensor de Reynolds, mostrando os possíveis estados da turbulência. Adaptado de [67].

Figura 40 - Valores do parâmetro $R$ no campo de medição. As regiões com maiores valores de $\boldsymbol{R}$ estão mais afastados do ponto de isotropia. Linhas de corrente mostradas em branco.

Figura 41 - Campo das invariantes de Lumley $(\bullet)$ e evolução longitudinal mostrando a tendência à isotropia $(\mathbf{\square}) \boldsymbol{X} / \boldsymbol{D} \boldsymbol{b}=\mathbf{0} \mathrm{e}$ $(\diamond) X / D b=-1$.

Figura 42 - Evolução do padrão de atomização conforme se incrementa a vazão de etanol (a) Caso D, (b) Caso E e (c) Caso F. (d) Ampliação da imagem instantânea do caso E. 
Figura 43 - Sequencia de imagens instantâneas mostrando a formação de gotas a partir dos ligamentos, ver região $(400,300)$. Lapso de tempo entre imagens $\boldsymbol{d t}=\mathbf{3} \boldsymbol{\mu} \boldsymbol{s}$.

Figura 44 - Evolução transversal do spray de etanol para o caso $\boldsymbol{E}$, para diferentes posições longitudinais (a) Número de gotas e (b) Diâmetro médio Sauter (SMD).

Figura 45 - Histograma da distribuição de diâmetros de gota para duas alturas a jusante do atomizador (a) $y=\mathbf{2 0} \mathbf{~ m m}$ e (d) $\boldsymbol{y}=$ $\mathbf{5 0} \mathbf{m m}$, ao longo de $\boldsymbol{x}=\mathbf{0}$.

Figura 46 - Dispersão da centricidade de gota para duas diferentes posições a jusante do atomizador (a) $\boldsymbol{y}=\mathbf{2 0} \mathbf{~} \mathbf{m m}$ e (b) $\boldsymbol{y}=\mathbf{5 0} \mathbf{~} \mathbf{m}$, ao longo de $\boldsymbol{x}=\mathbf{0}$.

Figura 47 - Histograma da distribuição de centricidade de gota para duas posições a jusante do atomizador (a) $\boldsymbol{y}=\mathbf{2 0} \mathbf{~} \mathbf{m m}$ e (b) $\boldsymbol{y}=\mathbf{5 0} \mathbf{~ m m}$, ao longo de $\boldsymbol{x}=\mathbf{0}$.

Figura 48 - Dispersão da componente longitudinal da velocidade das gotas, $\boldsymbol{V} \boldsymbol{y}$, para duas posições a jusante do atomizador (a) $\boldsymbol{y}=\mathbf{2 0} \mathbf{m m}$ e (b) $\boldsymbol{y}=\mathbf{5 0} \mathbf{m m}$, ao longo de $\boldsymbol{x}=\mathbf{0}$.

Figura 49 - Dispersão da componente transversal da velocidade das gotas, $\boldsymbol{V} \boldsymbol{x}$, para duas posições a jusante do atomizador (a) $\boldsymbol{y}=\mathbf{2 0} \mathbf{m m}$ e (b) $\boldsymbol{y}=\mathbf{5 0} \mathbf{m m}$, ao longo de $\boldsymbol{x}=\mathbf{0}$.

Figura 50 - Comparação das características de atomização dos casos $\boldsymbol{B}$ e $\boldsymbol{E}$ (a) Número de gotas e (b) Diâmetro médio Sauter (SMD), ao longo de $y=\mathbf{2 0} \mathbf{~ m m}$.

Figura 51 - Comparação do histograma da distribuição de diâmetros de gota em $\boldsymbol{x}=\mathbf{1 0} \mathbf{~ m m}$. (a) Caso $\boldsymbol{B}$ e (d) Caso $\boldsymbol{E}$.

Figura 52 - Comparação da dispersão da componente longitudinal da velocidade, $\boldsymbol{V} \boldsymbol{y}$, em $\boldsymbol{x}=\mathbf{1 2}, \mathbf{5} \mathbf{m m}$. (a) Caso $\boldsymbol{B}$ e (b) Caso $\boldsymbol{E}$.

Figura 53 - Fotografias das chamas turbulentas de spray de etanol, obtidas para uma velocidade de escoamento de $\operatorname{ar} \boldsymbol{V a}=\mathbf{8}, \mathbf{0} \mathrm{m} / \mathrm{s}$ (a) Caso A (b) Caso B e (c) Caso C. 
Figura 54 - Fotografias das chamas turbulentas de spray de etanol, obtidas para uma velocidade de escoamento de ar $\boldsymbol{V a}=\mathbf{1 1}, \mathbf{8} \mathrm{m} / \mathrm{s}$ (a) Caso $\boldsymbol{D}$ (b) Caso $\boldsymbol{E}$ e (c) Caso $\boldsymbol{F}$.

Figura 55 - Imagens instantâneas de PLIF-OH no caso $A$, superpostas ao sinal Mie (esquerda) e ao campo de velocidade das gotas (direita). $(V a=8,0 \mathrm{~m} / \mathrm{s}, \mathrm{Ree}=3,89 \times 103$ ).

Figura 56 - Imagens instantâneas de PLIF-OH no caso $D$, superpostas ao sinal Mie (esquerda) e ao campo de velocidade das gotas (direita). $(V a=11,8 \mathrm{~m} / \mathrm{s}, \mathrm{Ree}=3,89 \times 103$ ).

Figura 57 - Imagens instantâneas de PLIF-OH no caso $\boldsymbol{B}$, superpostas com a sinal Mie (esquerda) e ao campo de velocidade das gotas (direita). ( $\boldsymbol{V a}=\mathbf{8}, 0 \mathrm{~m} / \mathrm{s}, \boldsymbol{R e e}=\mathbf{5}, \mathbf{8 4 \times 1 0 3})$.

Figura 58 - Imagens instantâneas de PLIF-OH no caso $E$, superpostas com a sinal Mie (esquerda) e ao campo de velocidade das gotas (direita). $\boldsymbol{V a}=\mathbf{1 1}, 8 \mathrm{~m} / \mathrm{s}, \mathrm{Ree}=\mathbf{5}, \mathbf{8 4 x 1 0 3}$.

Figura 59 - Superposição das imagens médias do PLIF-OH e espalhamento Mie para uma vazão de etanol de $2,25 \mathrm{~cm} 3 / \mathrm{s}$ (a) Caso $\boldsymbol{A}$ e (b) Caso $\boldsymbol{D}$.

Figura 60 - Superposição das imagens médias do PLIF-OH e espalhamento Mie para uma vazão de etanol de $3,37 \mathrm{~cm} 3 / \mathrm{s}$ (a) Caso $\boldsymbol{B}$ e (b) Caso $\boldsymbol{E}$.

Figura 61 - Campo de velocidade média, $\boldsymbol{V} \boldsymbol{y}$, de gotas e vetores velocidade para uma vazão de etanol de $2,25 \mathrm{~cm} 3 / \mathrm{s}$ (a) Caso $A$, (b) Caso $\boldsymbol{D}$.

Figura 62 - Campo de velocidade média, $\boldsymbol{V} \boldsymbol{y}$, de gotas e vetores velocidade para uma vazão de etanol de $3,37 \mathrm{~cm} 3 / \mathrm{s}$ (a) Caso $B$, (b) Caso $\boldsymbol{E}$.

Figura 63 - Componente longitudinal de velocidade, $\boldsymbol{V y}$, para os casos $\boldsymbol{A}$ e $\boldsymbol{D}$ (a) linha $\boldsymbol{x}=\mathbf{0} \mathbf{m m}$ e (b) evolução transversal a diferentes alturas a jusante do atomizador. Vazão de etanol de $2,25 \mathrm{~cm} 3 / \mathrm{s}$.

Figura 64 - Componente longitudinal de velocidade, $\boldsymbol{V} \boldsymbol{y}$, para os casos $\boldsymbol{B}$ e $\boldsymbol{E}$ (a) linha $\boldsymbol{x}=\mathbf{0} \boldsymbol{m m}$ e (b) evolução transversal a 
diferentes alturas a jusante do atomizador. Vazão de etanol de $3,37 \mathrm{~cm} 3 / \mathrm{s}$.

Figura 65 - Orifício do atomizador medido com microscópio.

Figura 66 - Evolução das componentes do tensor de Reynolds na direção $(\mathrm{x})$ no caso 1 para diferentes alturas acima do obstáculo (a) $R x x V y \_m a x 2$, (b) RyyVy_max2, (c) RzzVy_max2.

Figura 67 - Evolução das componentes do tensor de Reynolds na direção $(\mathrm{x})$ no caso 1 para diferentes alturas acima do obstáculo (a) RxxVy_max2, (b) RyyVy_max2, (c) RzzVy_max2, (d) $R x y V y_{-} \max 2$, (e) $R x z V y \_\max 2$ e (f) RyzVy_max2.

Figura 68 - Evolução das componentes do tensor de Reynolds na direção $(\mathrm{x})$ no caso 2 para diferentes alturas acima do obstáculo (a) RxxVy_max2, (b) RyyVy_max2, (c) RzzVy_max2.

Figura 69 - Evolução das componentes do tensor de Reynolds na direção (x) no caso 2 para diferentes alturas acima do obstáculo (a) $R x x V y \_\max 2$, (b) RyyVy_max2, (c) RzzVy_max2, (d) $R x y V y_{-} \max 2$, (e) $R x z V y_{-} \max 2$ e (f) $R y z V y_{-} \max 2$. Figura 70 - Resultados da medição do diâmetro e centricidade dos círculos da placa de calibração da profundidade de campo. 


\section{Lista de tabelas}

Tabela 1 - Características nominais do spray resultante utilizando água [29].

Tabela 2 - Propriedades do etanol hidratado [31,32].

Tabela 3 - Espécies químicas frequentemente usadas em

aplicações de LIF para avaliar características das chamas [35].

Tabela 4 - Parâmetros dos experimentos de escoamento anular de ar.

Tabela 5 - Parâmetros dos escoamentos de ar e etanol

empregados nos testes sem e com combustão de etanol.

Tabela 6 - Valores de incerteza da velocidade do spray de etanol para as vazões estudadas.

Tabela 7 - Valores de incerteza das variáveis empregadas no calculo do numero de Reynolds da injeção de etanol.

Tabela 8 - Máximas incertezas estimadas para os círculos da placa de calibração da profundidade de campo. 


\section{Lista de Símbolos}

\section{Letras Latinas}

$A_{10} \quad$ Coeficiente de Einstein da taxa relativa de emissão espontânea $\left(s^{-1}\right)$.

$B \quad$ Constante de Boltzman.

$b_{i j} \quad$ Tensor anisotrópico.

c Centricidade

$D_{10} \quad$ Diâmetro médio aritmético.

$D_{32} \quad$ Diâmetro médio Sauter $(\mu \mathrm{m})$.

$D_{v 50} \quad$ Diâmetro volumétrico médio.

$D_{b} \quad$ Diâmetro do obstáculo bluff-body $(\mathrm{mm})$.

$d t \quad$ Lapso de tempo entre dois pulsos laser $(s)$.

d Diâmetro de gota $(\mu m)$.

$d_{b} \quad$ Diâmetro da boquilha do atomizador $(\mu m)$.

$d_{p} \quad$ Diâmetro da partícula $(\mu m)$.

${ }^{\circ} G L \quad$ Grau Gay Lussac.

$I_{t} \quad$ Intensidade turbulenta.

$\dot{m} \quad$ Vazão mássica $(\mathrm{kg} / \mathrm{s})$.

$N \quad$ Número de gotas.

$N^{\prime} \quad$ Número de gotas corregidas.

$p \quad$ Pressão (bar).

ppm Partículas por milhão.

$R e_{a} \quad$ Número de Reynolds do escoamento de ar.

$R e_{j} \quad$ Número de Reynolds do spray de etanol.

$R_{i j} \quad$ Tensor de Reynolds.

$S_{i j} \quad$ Tensor de deformação.

$V_{a} \quad$ Velocidade longitudinal do ar.

$V_{y} \quad$ Velocidade longitudinal.

$V_{x} \quad$ Velocidade transversal. 


$\begin{array}{ll}x & \text { Direção transversal (sentido radial do queimador). } \\ y & \text { Direção vertical (coincidente com a linha de simetria do } \\ & \text { queimador). } \\ W_{i j} & \text { Tensor vorticidade. } \\ W e & \text { Número de Weber do spray de etanol. } \\ \lambda & \text { Comprimento de onda }(\mathrm{nm}) . \\ v_{t} & \text { Viscosidade turbulenta. } \\ \delta_{i j} & \text { Delta de kronecker. } \\ \rho & \text { Densidade }\left(\mathrm{kg} / \mathrm{m}^{3}\right) . \\ \partial & \text { Derivada parcial. }\end{array}$

\section{Subscritos}

$\begin{array}{ll}a & \text { Ar. } \\ e & \text { Etanol. } \\ \text { inj } & \text { Injetor ou injeção. }\end{array}$

\section{Siglas}

CCD Charged coupled device.

EHC Etanol hidratado combustível.

ICCD Intensificador charged coupled device.

IMI Interferometry Mie.

IRO Intensified Relay Optics.

LIF Fluorescência induzida por laser.

LDV Laser Doppler Velocimetry.

Mie Espalhamento Mie.

$\mathrm{MCl} \quad$ Motores de combustão interna.

Nd:YAG Laser de estado solido de Neodímio $\left(N d^{3+}\right)$ e ítrio de alumínio $\left(\mathrm{Y}_{3} \mathrm{Al}_{5} \mathrm{O}_{12}\right)$.

PDA Phase Doppler Anemometer.

PFI Port fuel injetor.

PIV Velocímetria por imagem de partículas.

PLIF Fluorescência induzida em um plano laser.

PLIEF Fluorescência exciplex induzida em um plano laser. 
PDF Probability density functions.

SMD Diâmetro médio Sauter.

TER Transferência de energia rotacional.

TEV Transferência de energia vibracional.

PTV Particle tracking velocimetry.

\section{Compostos químicos}

CO Monóxido de carbono.

$\mathrm{CO}_{2} \quad$ Dióxido de carbono.

DEMA Diethylmethylamide.

FB Fluorobenzeno.

$H \quad$ Hidrogênio atômico.

$\mathrm{H}_{2} \mathrm{O} \quad$ Agua.

NO Monóxido de nitrogênio.

$\mathrm{NO}_{2} \quad$ Óxido nítrico.

$\mathrm{OH} \quad$ Hidroxila.

$C_{2} \quad$ Carbono molecular.

$\mathrm{O}_{2} \quad$ Oxigênio.

$\mathrm{N}_{2} \quad$ Nitrogênio.

$\mathrm{TiO}_{2} \quad$ Dióxido de Titânio.

$M \quad$ Monômero.

$N \quad$ Formador do composto exciplex. 